\title{
Principal stress behaviour of a steel plate shear wall concerning buckling modes
}

\author{
P. Memarzadeh, M. Azhari \& M. M. Saadatpour \\ Department of Civil Engineering, Isfahan University of Technology, Iran
}

\begin{abstract}
When buckling occurs in the infill plate of a steel plate shear wall (SPSW), a diagonal tension field is formed through the plate. This paper investigates the influence of torsional stiffness of surrounding members (i.e. beams and columns) on the buckling coefficient and tension field behaviour of SPSW. The linear buckling equations in the sense of von-Karman have been solved in conjunction with various boundary conditions, by using the Ritz method. Also, in this research the effects of symmetric and anti-symmetric buckling modes on the behaviour of the tension field and buckling coefficient have been studied.

Keywords: steel shear wall, thin plate, shear buckling, symmetric, antisymmetric, Ritz method, principal stresses.
\end{abstract}

\section{Introduction}

The steel plate shear wall is a lateral load resisting system consisting of an infill plate located within a frame. While performing experimental investigations on the thin aluminum shear panels of an aircraft, Wagner found out that in thinwebbed structures with stiff boundary members a diagonal tension field would be formed when buckling occurs. Then Wagner [1] developed the pure tension theory stating that the formation of the tension field is the primary mechanism for shear resistant. The incomplete tension field theory was later presented by Kuhn et al. [2]. On the basis of Kuhn's theory the shear resistance capacity is a combination of pure shear and inclined tension field.

Design engineers require the ability to assess inelastic structural response using conventional analysis software that is commonly available. An analytical model-termed the strip model-was developed by Thorburn et al. [3] to simulate the tension field behaviour, wherein the infill plate is modelled as a 
series of tension-only strips at the same angle of inclination, $\theta$, as the tension field. They derived the angle of inclination for the strips, $\theta$, from the principle of Least Work as a function of axial stiffness of boundary members.

By including the effect of in-plane flexural stiffness of boundary members and employing the principle of Least Work, Timler and Kulak [4] derived another equation for $\theta$ in terms of axial and flexural rigidities of surrounding members.

The Canadian Steel Design Standard [5] suggests the application of the strip model as a design tool for steel plate shear wall (CAN/CSA 516-01) and the equation derived by Timler and Kulak [4] for the calculation of $\theta$ (clause 20.3.1). However, researchers are still searching for an increase in the precision of the prediction of the overall behavior of the shear wall.

This paper investigates the effect of different parameters on buckling loads as well as on the distribution and orientation patterns of the tension field principal stresses. These parameters include torsional stiffness of boundary members as well as symmetric and anti-symmetric buckling modes.

\section{Theory}

\subsection{Modelling of SPSW}

The surrounding members of the SPSW are modeled by the springs.

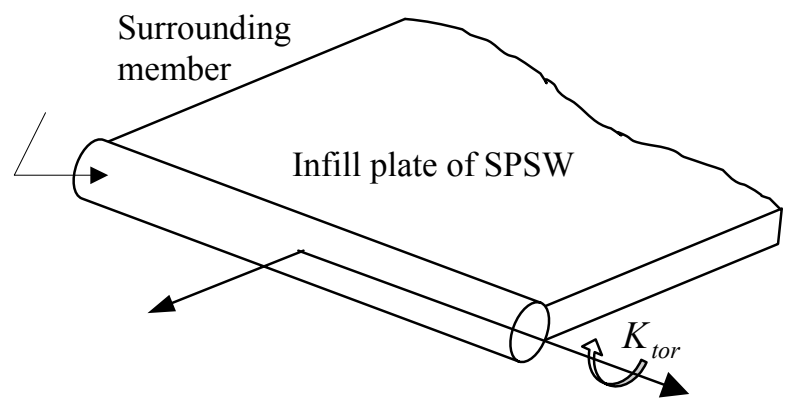

Figure 1: $\quad$ General scheme for a section of the model.

To define logical parameter for the amount of torsional stiffness of surrounding members, the non-dimensional stiffness parameter $\alpha$ is introduced as follows:

$$
\alpha=\frac{K_{t o r}}{D}
$$


where $K_{\text {tor }}$ is the unit length torsional stiffness of surrounding members and $D$ is the flexural rigidity of plate. A model is defined for studying the effect of the stiffness parameter $\alpha$ shown in fig. 1. This way, a comparison between the effectiveness of different stiffness parameters of surrounding members is carried out.

\subsection{Ritz method}

This paper utilizes the Ritz method to analyze the buckling of infill plate of a SPSW under an applied in-plane shear loading (fig. 2). The geometric $\left(V_{p}\right)$ and the elastic strain energy $(U)$ are the variants used in the energy solution, and are given by the following equations:

$$
V_{p}=-N_{x y} \int_{A}\left(w_{, x} w_{, y}\right) d A
$$

where $N_{x y}, A$ and $w$ are the elastic shear buckling load, the area and the lateral buckling displacement of the plate, respectively. The comma denotes differentiation with respect to the corresponding co-ordinates.

$$
U=\frac{D}{2} \int_{A}\left[\left(w_{, x x}+w_{, y y}\right)^{2}-2(1-v)\left(w_{, x x} w_{, y y}-w_{, x y}^{2}\right)\right] d A+U_{s}
$$

in which $U_{s}$ is the strain energy of the spring are defined by:

$$
\begin{aligned}
U_{s}= & \left.\int \frac{K_{\text {tor }}}{2}\left(\left.w_{, x}^{2}\right|_{x=-\frac{a}{2}}+\left.w_{, x}^{2}\right|_{x=\frac{a}{2}}\right)\right] d y \\
& +\int\left[\frac{K_{\text {tor }}}{2}\left(\left.w_{, y}^{2}\right|_{y=-\frac{b}{2}}+\left.w_{, y}^{2}\right|_{y=\frac{b}{2}}\right)\right] d x
\end{aligned}
$$

In the use of the Ritz method, an appropriate displacement function for $w$ must be chosen. That used herein is the polynomial-based displacement function which consists of a boundary polynomial specifying the geometric and kinematic boundary conditions multiplied by a complete simple polynomial. This displacement function is written by:

$$
w=\varphi_{b}(\xi, \eta) \sum_{q=0}^{p} \sum_{r=0}^{q} a_{m} \phi_{m}(\xi, \eta)
$$

where $p$ is the degree of a two-dimensional polynomial and $a_{m}$ is the arbitrary Ritz coefficient. 


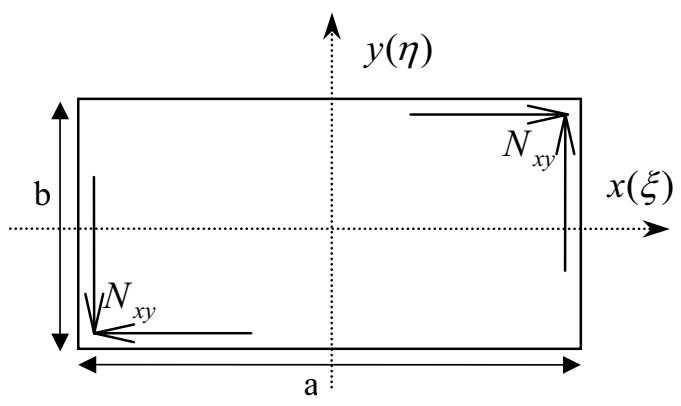

Figure 2: $\quad$ Isotropic plate under pure shear.

$\phi_{m}(\xi, \eta)$ is the m-th term of a two-dimensional polynomial as below (Smith et al. [6]):

$$
\phi_{m}(\xi, \eta)=\xi^{r} \eta^{q-r}
$$

in which $\xi=2 x / a, \eta=2 y / b$ is given by:

$$
m=\frac{(q+1)(q+2)}{2}-r
$$

The term $\varphi_{b}(\xi, \eta)$ is the boundary polynomial describing the boundary conditions defined by:

$$
\varphi_{b}(\xi, \eta)=(\xi-1)^{1}(\xi+1)^{1}(\eta-1)^{1}(\eta+1)^{1}
$$

In the buckling analysis, the kinematic and geometric boundary conditions are specified when the boundary polynomial $\varphi_{b}(\xi, \eta)$ is multiplied by the corresponding internal interpolation polynomial.

\subsection{Linear eigenvalue analysis}

The total potential energy $\Pi$ of the system is given by:

$$
\Pi=U+V_{p}
$$

Based on the principal of minimum potential energy, the total potential $\Pi$ in eqn. (9) is minimized with respect to the unknown Ritz coefficient $a_{m}$. Because $\Pi$ is a function of the product of Ritz coefficients $a_{m} a_{n}$, minimization by 
formal differentiation leads to a set of simultaneous linear independent equations. The solution of these equations produced the eigenvalues (buckling loads) and substituting of the corresponding eigenvectors into the displacement function $w$ in eqn. (5) as the Ritz coefficients gives the buckling modes.

\subsection{Stress analysis}

Since the buckling modes of a plate specify the proportional values of transverse deflections, the corresponding values of strains and stresses will be calculated proportionally. Using the transverse deflection $w$, the stresses in the mid-plane of plate can be written by:

$$
\begin{aligned}
\sigma_{x} & =\frac{E}{2}\left(w_{, x}{ }^{2}+v w_{, y}{ }^{2}\right) \\
\sigma_{y} & =\frac{E}{2}\left(w_{, y}{ }^{2}+v w_{, x}{ }^{2}\right) \\
\tau_{x y} & =G w_{, x} w_{, y}
\end{aligned}
$$

where $G$ is the shear modulus of elasticity. Using the Mohr's circle, the state of stresses can be represented in the principal coordinates. Also the angle of inclination of the tension field can be calculated by determining the orientation of the principal stresses. Then, it is possible to plot the distribution and orientation patterns of the principal stresses in the tension field of a plate.

\section{Numerical parametric studies}

\subsection{Shear buckling analysis}

A computer program has been developed based on the von-Karman theory and the Ritz method. The numerical analyses were performed by the computer program. In these buckling analyses, the value of $p$ was selected equal to 8 . To compare the various buckling analyses, the non-dimensional buckling coefficient was employed as follows:

$$
k_{s}=\frac{N_{x y} b^{2}}{\pi^{2} D}
$$

By plotting the various buckling mode shapes, it will be specified which modes are symmetric or anti-symmetric. The "first" symmetric and anti-symmetric modes are corresponding with the minimum values of the symmetric and antisymmetric buckling loads, respectively. However, in this paper the word "first" is omitted for brevity. On the purpose of verifying the validity of buckling analyses, the results are compared with the available references. So, the stiffness 
of spring is selected equal to zero or infinite for modelling simply support (S) or clamped edges $(\mathrm{C})$, respectively. In table 1 , the resulting buckling coefficients from the present analyses have been compared with those reported in references. As the table 1 shows, the results are in good agreement.

Table 1: Comparison the present results with those of available reference.

\begin{tabular}{|c|c|c|c|c|c|}
\hline \multirow{3}{*}{$\begin{array}{l}\text { Stiffness } \\
\text { Parameter }\end{array}$} & \multirow{3}{*}{$\begin{array}{l}\text { Boundary } \\
\text { Conditions }\end{array}$} & \multirow{3}{*}{$a / b$} & \multicolumn{3}{|c|}{$k_{s}$} \\
\hline & & & \multicolumn{2}{|c|}{ Present } & \multirow{2}{*}{$\begin{array}{c}\text { Timoshenko } \\
\text { (1963) }\end{array}$} \\
\hline & & & Symmetric & $\begin{array}{c}\text { Anti- } \\
\text { symmetric }\end{array}$ & \\
\hline \multirow{4}{*}{$\alpha=0$} & \multirow{4}{*}{ SSSS } & 1.0 & 9.3254 & 11.5484 & 9.34 \\
\hline & & 1.5 & 7.0707 & 7.9591 & 7.10 \\
\hline & & 2.0 & 6.5464 & 6.5781 & 6.60 \\
\hline & & 3.0 & 5.9535 & 5.8465 & 5.90 \\
\hline \multirow{4}{*}{$\alpha=\infty$} & \multirow{4}{*}{ CCCC } & 1.0 & 14.6515 & 17.1165 & 14.71 \\
\hline & & 1.5 & 11.4791 & 12.0293 & 11.50 \\
\hline & & 2.0 & 10.6527 & 10.5545 & 10.34 \\
\hline & & 3.0 & 9.8449 & 10.6985 & ------- \\
\hline
\end{tabular}

Typically, the symmetric and anti-symmetric buckling modes of plate are depicted in three-dimension views (fig. 3). Fig. 4 shows the effect of varying the stiffness parameters $\alpha$ on the symmetric and anti-symmetric buckling coefficients of plate. The following results can be concluded by attending to these figures:

- The symmetric and anti-symmetric buckling coefficients of a plate with aspect ratio equal or greater than 1.5 are close together.

- Although the symmetric buckling mode is often the critical mode of shear buckling, sometimes the anti-symmetric mode would be critical.

- Fig. 4 shows that the shear buckling mode of a plate would not be changed by varying the stiffness parameter $\alpha$; because there is no intersection for curves in fig. 4.

\subsection{Stress analysis}

\subsubsection{Principal stress distribution pattern (PSDP)}

By comparing the PSDPs with the corresponding buckling modes, the areas where the amounts of principal stresses are peak, may be specified. Fig. 5 illustrates these comparisons for two extreme values of zero and infinite for the stiffness parameter $\alpha$. This figure shows that the peak(s) of principal stresses occurs at the slope(s) of buckling mode shapes for both symmetric and antisymmetric modes. Therefore, in symmetric buckling modes, the principal stresses peaks are being at both sides of the plate centre, while in anti-symmetric buckling this peak would be in centre of the plate. Also, fig. 5 shows that the PSDPs are symmetric for both symmetric and anti-symmetric buckling modes. 


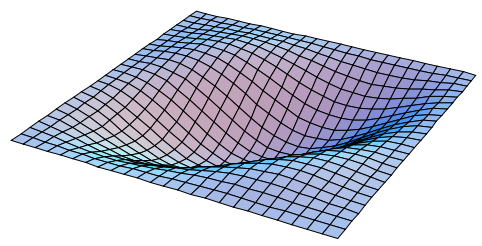

(a)

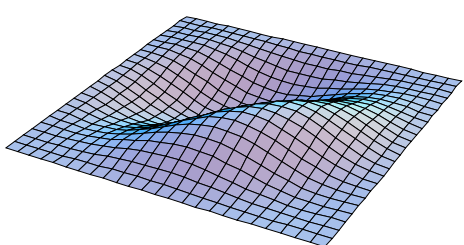

(b)

Figure 3: $\quad$ Symmetric (a) and anti-symmetric (b) shear buckling modes.

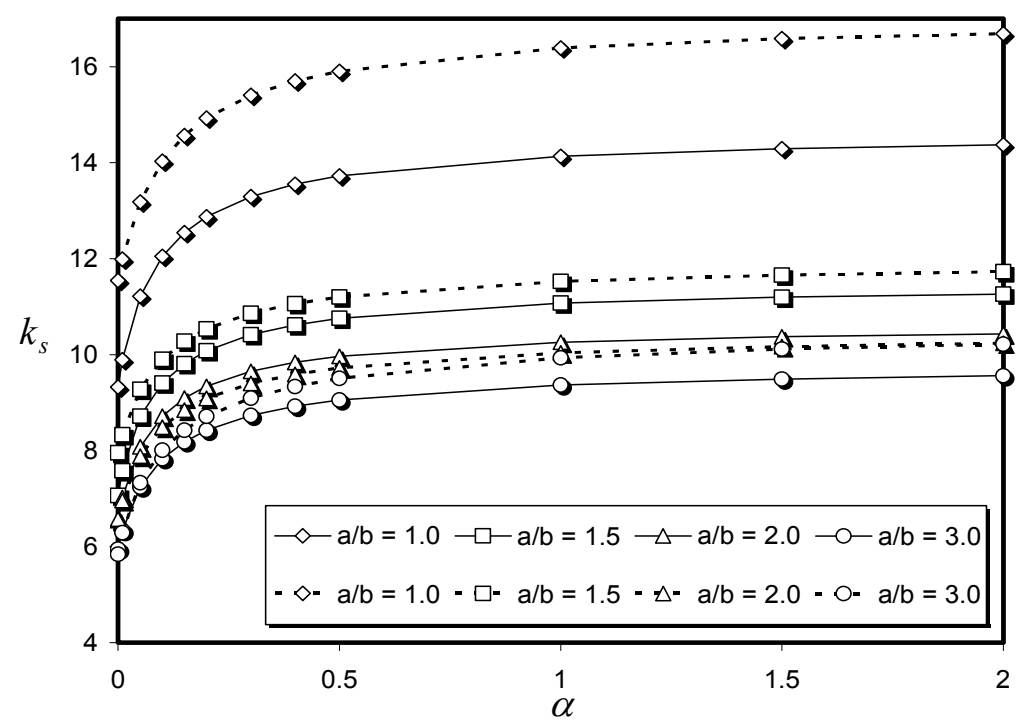

Figure 4: $\quad$ Shear buckling coefficient vs. $\alpha$ (line for symmetric and dashed for anti-symmetric buckling).

\subsubsection{Principal stress orientation pattern (PSOP)}

For showing some patterns simultaneously, it is advantageous that the patterns are putted together and combined as shown in fig. 6. The orientations of principal stresses can be determined at each point of the plate by using the Mohr's circle. Fig. 7 shows the combined PSOPs related to various values of stiffness parameters $\alpha$. In this figure, the orientation of each depicted line represents the orientation of the related principal stress. By careful observation, it is realized that, there are areas in the plate where the orientations of related principal stresses will not be changed by varying the value of the stiffness parameter $\alpha$. 


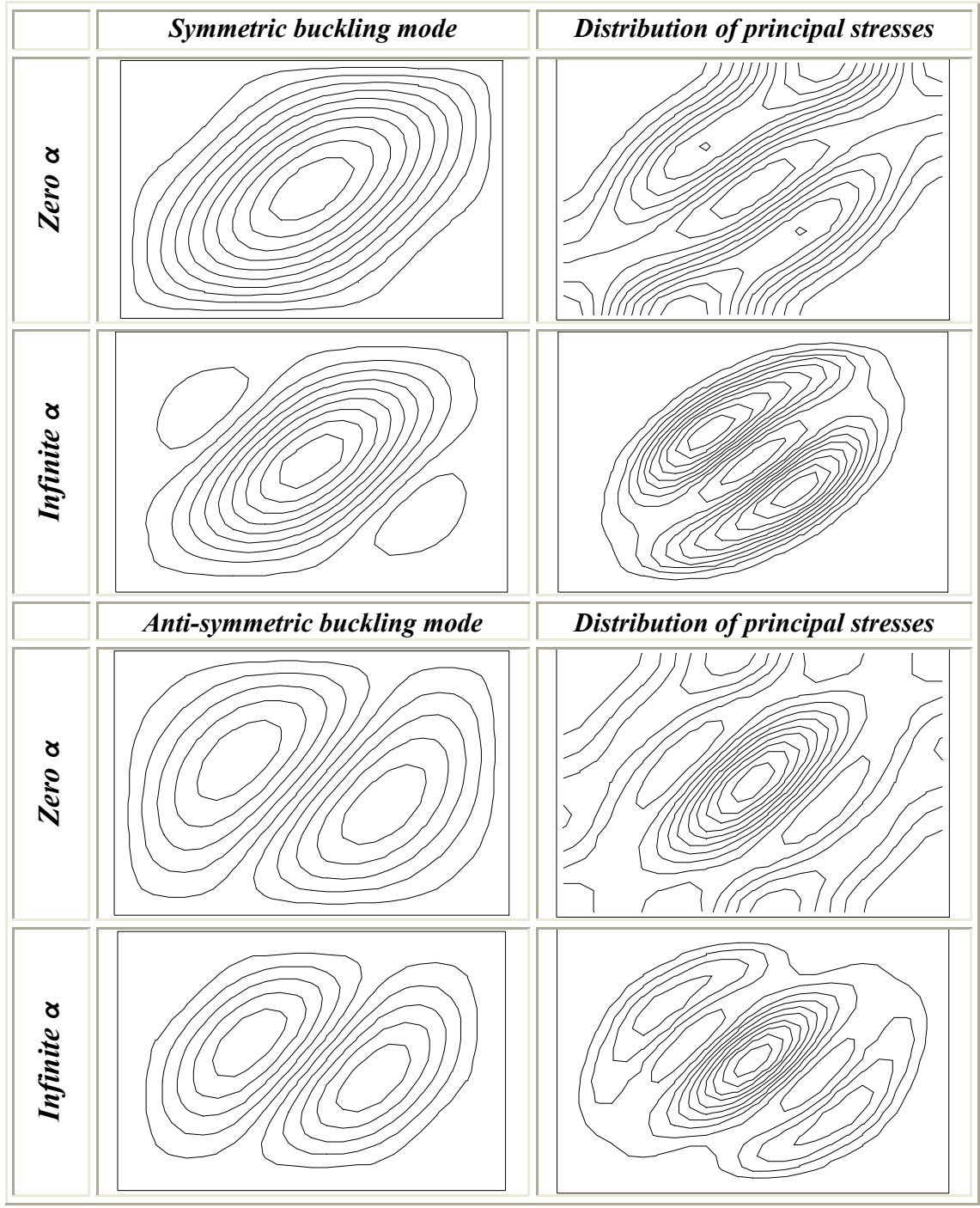

Figure 5: $\quad$ Symmetric and anti-symmetric buckling mode shapes and PSDPs for two extremes of $\alpha=0$ and $\alpha=\infty$ (aspect ratio 1.5).

These areas of the plate in symmetric buckling are more extended than those in anti-symmetric buckling. Also, these areas have different distribution for the symmetric and anti-symmetric buckling modes.

Fig. 8 shows the combinations of PSOPs related to symmetric and antisymmetric buckling modes. This figure reveals that the PSOPs are relatively different for the symmetric and anti-symmetric buckling modes. 

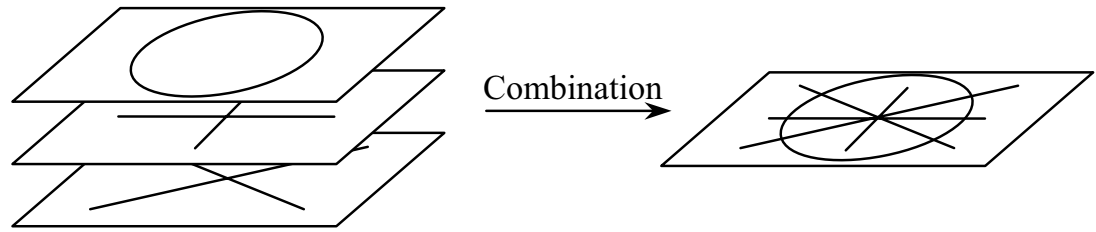

Figure 6: Scheme for combination of some patterns.

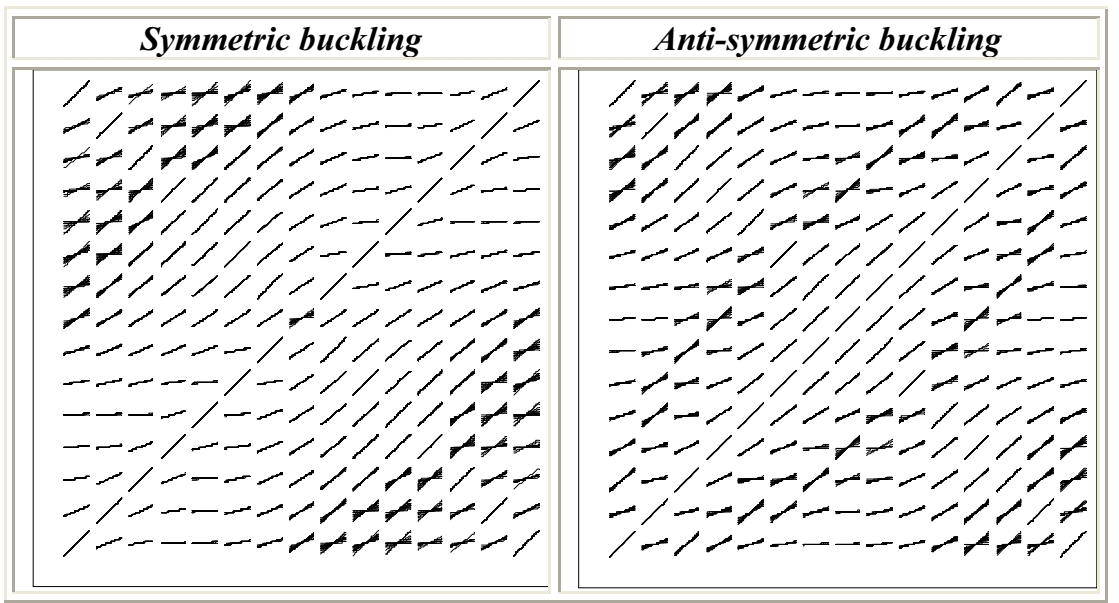

Figure 7: Combination of PSOPs related to various $\alpha$ for symmetric and antisymmetric buckling modes.

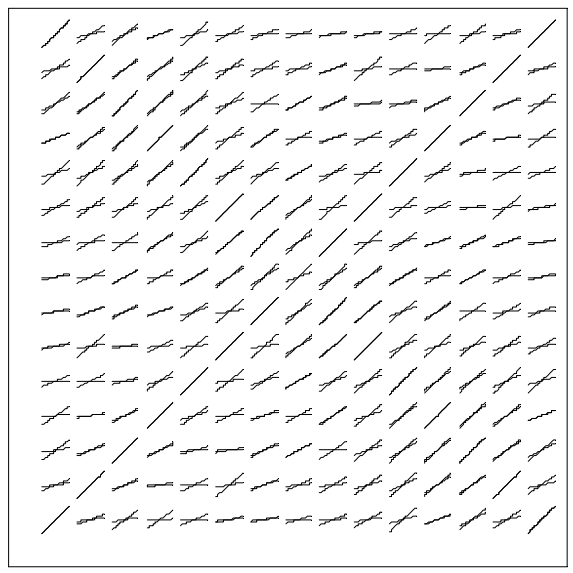

Figure 8: Combination of PSOPs related to symmetric and anti-symmetric buckling modes $(\alpha=0)$. 


\section{Conclusions}

Initial imperfection of plates due to their fabrication causes that the plates do not experience the buckling bifurcation point. The type of postbuckling mode of a plate may be a function of its initial imperfection, especially for the plate with close buckling loads correspond with different buckling modes. This research reveals that buckling loads correspond with first symmetric and anti-symmetric buckling modes of a plate with an aspect ratio equal or greater than 1.5 are close together. This result specifies the important role of the initial imperfection of a plate in determining the postbuckling mode of the plate.

It is also shown that the orientation patterns of principal stresses correspond with the symmetric and anti-symmetric buckling modes of a plate are different, relatively. Since the angle of inclination of the tension field of a SPSW is an effective parameter on development of the strip model, so, this result may be vital in modifying the strip model. The role of initial imperfection of the plate in determining the type of buckling mode has not been included in any analytical models presented so far.

These studies also reveal that variation of amount of torsional stiffness of boundary members does not change the orientations of principal stresses in some areas of the plate. These areas where located in the slopes of the buckling mode shapes have relatively great principal stresses.

\section{References}

[1] Wagner, H., Flat sheet metal girders with very thin webs, Part I - General theories and assumptions. Technical Memo No. 604, National Advisory Committee for Aeronautics, Washington, D.C, 1931.

[2] Kuhn, P., Peterson, J.P., and Levin, L.R., A summary of diagonal tension, part I - Methods of analysis. Technical Note 2661, National Advisory Committee for Aeronautics, Washington, D.C, 1952.

[3] Thorburn, L.J., Kulak, G.L., and Montgomery, C.J., Analyses of steel plate shear walls. Structural Engineering Report No. 107, University of Alberta, Canada, 1983.

[4] Timler, P.A., Kulak, G.L., Experimental study of steel plate shear walls, Structural Engineering Report No. 114, Department of Civil Engineering, University of Alberta, Edmonton, Canada, 1983.

[5] Canadian Standard Association, CAN/CSA S16-01, Limit States Design of Steel Structures, Toronto, Ontario, 2001.

[6] Smith, S.T., Bradford, M.A., and Oehlers, D.J., Elastic buckling of unilaterally constrained rectangular plates in pure shear. Engineering Structures, 21, pp. 443-453, 1999. 